\title{
The effect of sugar and artificial sweetener on molecular markers of metabolic syndrome: a mice study
}

\author{
${ }^{1}$ Subali, D., ${ }^{2}$ Silo,W., ${ }^{1}$ Listyani, L., ${ }^{1}$ Endriani, C., ${ }^{2, *}$ Kartawidjajaputra, F. and \\ ${ }^{1}$ Suwanto, A. \\ ${ }^{1}$ Department of Biotechnology, Atma Jaya Catholic University, Jakarta, 12930, Indonesia \\ ${ }^{2}$ Nutrifood Research Center, PT Nutrifood Indonesia, Jakarta, 13920, Indonesia
}

\begin{abstract}
Article history:
Received: 16 May 2017

Received in revised form:

21 June 2017

Accepted: 24 June 2017

Available Online: 24 June 2017
\end{abstract}

\section{Keywords:}

Aspartame,

fto,

Molecular markers,

C. coccoides

\section{DOI:}

http://doi.org/10.26656/ fr.2017.6.052

\begin{abstract}
The usage of aspartame, as one of the most widely used sweetener, has been approved in many types of food products. Moreover, many studies have proven that replacing sugar with aspartame would contribute favorable effects on several health parameters; such as, body weight, blood glucose level, and inflammatory status. In this experiment, we examined the effects of aspartame consumption on some biomarkers; which potentially acted as early signals for a personal metabolic status. This study was aimed to investigate the effect of aspartame on the expression of a number of molecular markers related with appetite regulation (fto), fat accumulation markers (fabp4 and alt2) and inflammation marker (tnf- $\alpha$ ) in Sprague Dawley rats. The population of Clostridium coccoides was also observed to give an insight about the effect of sweetener consumption on gut microbiota profiles. 15 healthy, male, eight-weeks old Sprague-Dawley rats were fed a standard diet and divided into 3 groups ( $\mathrm{n}=5$ for each): water only, sucrose $(30 \% \mathrm{~b} / \mathrm{v})$, and aspartame $(0.15 \% \mathrm{~b} / \mathrm{v})$. Body weight was measured weekly and blood glucose measurement was carried out on day 1 and 40 . At the end of the experiment, all rats were euthanized and blood was collected from the vein. The liver, brain, and visceral adipose tissue were excised, weighed, and grinded with liquid nitrogen. Feces samples were collected on day 0 and 40. At the end of our experimental period; the body weight, liver weight, and blood glucose level of sucrose-treated rats were significantly higher $(\mathrm{p}<0.05)$ than aspartame and control group. Sucrose showed the lowest level of fto gene expression; yet, the fto gene expression in aspartame group was still lower than the control group. Expression of several genes considered as metabolic syndrome-related biomarkers were measured (fabp4, alt2, and tnf- $\alpha$ ); and our data demonstrated that sucrose treatment gave the highest increase in expression level of those genes; while aspartame treatment showed much lower values. Furthermore, sucrose also caused a significant reduction in C. coccoides population; while, the $C$. coccoides population in aspartame group did not differ significantly compared to the control group.
\end{abstract}

\section{Introduction}

Artificial sweeteners have been used for years as a sugar substitute for their "less calorie" property which leads to a healthier lifestyle and reduced metabolic syndrome rates. Aspartame, as one of the most widely used artificial sweetener, was claimed as a safe ingredient and would cause no harm as long as the consumption did not exceed the acceptable daily intake (ADI) based on
United States Food and Drug Administration (USFDA) and European Food Safety Authority (EFSA). However, several recent studies indicated some unfavorable effects of consuming aspartame.

A research demonstrated that aspartame could decrease some inflammation parameters, such as tumor necrosis alpha (TNF- $\alpha$ ), interleukin-2 (IL-2), and interferon gamma (IFN- $\gamma$ ), which were also used as markers of type 2 diabetes 
mellitus development. In contrary, this research also mentioned that aspartame acted as a chemical stressor and raised oxidative stress leading to inflammation (Choudhary and Devi 2015). Other studies also demonstrated that aspartame consumption could cause an increment of fat and body mass (Collison et al., 2012; Feijo et al., 2013), liver damage, and non-alcoholic fatty liver disease (NAFLD) (Abhilash et al., 2011). A study by Yang (2010) proposed that the sweet response produced after consuming artificial sweetener could increase the appetite and reduce satiety, which finally led to obesity development. One cohort study with a very large number of subjects also showed the increment of waist circumference after consuming diet coke containing artificial sweetener (Fowler et al., 2015).

To our knowledge, there was only very limited studies which investigate the effect of sweeteners on molecular markers related to appetite regulation, adiposity, and inflammation. Research were usually focused on parameters such as body weight, waist circumference, blood glucose and such; yet the influence on other surrogate biomarkers was not often explored. Thus, we would like to assess several promising markers, which potentially act as earlier signals.

Fto is a regulator protein which is produced in many organs, including hypothalamus; implying it plays a role in appetite regulation (Vujovic et al., 2013). Tumor necrosis factor- $\alpha$ (TNF- $\alpha)$ is a pro-inflammatory cytokine produced in blood, which initiates the immune response (Kempf et al., 2007). The high expression level of TNF- $\alpha$ is often found in subjects with metabolic disorder diseases like obesity and diabetes (Kempf et al., 2007; De Koning et al., 2012; Jung and Choi 2014; Choudhary and Devi 2015). Fabp4 is mainly expressed in adipose tissue and its expression is up-regulated during adipogenesis; hence it is often used as adipogenesis marker (Simon et al., 2013; Furuhashi et al., 2014). Alt2 is gluconeogenic enzymes which expressed mainly in the liver and frequently used as a marker for NAFLD development. A high expression of Alt2 also indicates a state of insulin resistance (Saltiel and Khan 2001).

Recently, the association of sweetener consumption with higher appetite was proposed to be mediated via shifting in gut microbiota population (Palmnas et al., 2014). Commensal Clostridia consist of Clostridium cluster XIVa and IV (Clostridium coccoides), which are known for years as fusiform-shaped bacteria; make up a substantial part (10-40\%) of the total bacteria in the gut microbiota. They are strongly involved in the maintenance of overall gut function. This leads to important translational implications in regard to the prevention and treatment of dysbiosis, to drug efficacy and toxicity, and to the development of therapies modulating the composition of the microflora (Lopetuso et al., 2013). Thus, it would be interesting to investigate how the sweetener consumption affects the Clostridia population. However, this is a new area of research and more studies are needed to obtain more convincing data.

Therefore, this study was aimed to investigate the effect of aspartame on several molecular markers related to appetite regulation (fto), fat accumulation marker (fabp4 and alt2) and inflammation marker (tnf- $\alpha$ ) in Sprague-Dawley rats. The population of Clostridium coccoides was also observed to give an insight into the effect of sweetener consumption on gut microbiota profile.

\section{Materials and methods}

\subsection{Materials}

RNase Zap was purchased from Ambion (Huntingdon, Cambridgeshire, United Kingdom), RNAprotect Animal Blood Tubes was purchased from Qiagen (Hilden, Germany), RNeasy Protect Animal Blood Kit was purchased from Qiagen (Hilden, Germany), RNeasy Lipid Tissue Mini Kit was purchased from Qiagen (Valencia, California, USA), RNeasy Plus Mini Kit was purchased from Qiagen (Valencia, California, USA), Allprotect Tissue Reagent was purchased from Qiagen (Valencia, California, USA), Revertaid First Strand cDNA Synthesis Kit was purchased from Thermo Fisher Scientific (Waltham, Massachusetts, United States), GoTaq Green Master Mix was purchased from Promega (Madison, Wisconsin, USA), SYBR Green PCR Master Mix was purchased from Applied Biosystem (Foster City, California,USA), tnf- $\alpha$ primer, fabp 4 primer, alt primer, fto primer and $\beta$ actin primer were purchased from IDT (Iowa, USA).

\subsection{Animal experiment}

This experiment was carried out by veterinarian from PT Bimana Indomedical (Bogor, Indonesia) after all protocols were approved by the Animal Welfare Supervision Commission and Use of Research Animals (ACUC number: R.04-14-IR). 15 healthy, male, eightweeks-old Sprague-Dawley rats weighing approximately 56.5-129.3 grams were fed a standard diet calculated based on rats' body weight and divided into 3 groups ( $\mathrm{n}=5$ for each group) based on the administered treatment: water only, sucrose $30 \% \mathrm{~b} / \mathrm{v}$, and aspartame $0.15 \% \mathrm{~b} / \mathrm{v}$. The amount of diet and water consumed were 
observed every day. Body weight was measured once a week for 40 days treatment and blood glucose measurement was carried out on day 1 and 40 .

\subsection{Sample collection}

At the end of the experiment, all rats were euthanized and blood was collected from the vein. Blood samples were transported to the analytical laboratory using RNAprotect Animal Blood Tubes and the other tissues were transported using Allprotect Tissue Reagent. Feces samples were collected on day 0 and day 40 during the intervention period. The liver, brain, and visceral adipose tissue were excised, weighed and grinded with liquid nitrogen, followed with homogenization using the buffer from RNA isolation kit.

\subsection{Blood glucose measurement}

Blood glucose was measured using GlucoDR (Hasuco Korea, Dongjakgu, Seoul, Korea). The tail's end of the rat was wounded to get the drop of blood for blood glucose measurement. The drop was dripped on the gluco-strip connected to the device. The number which appeared on the device was the blood glucose value.

\subsection{RNA isolation}

RNA isolation from adipose tissue was performed using RNeasy Lipid Tissue Mini Kit; RNA isolation from liver and brain was performed using RNeasy Plus Mini Kit; while RNA isolation from whole blood was performed using RNeasy Protect Animal Blood Kit. All the procedures were completed according to the manufacturer's protocol. The amount of samples used was $\pm 20 \mathrm{mg}$ for liver and $\pm 100 \mathrm{mg}$ for adipose and brain tissue. The blood samples' volume for RNA isolation was $200 \mu \mathrm{L}$.

\subsection{DNA total extraction}

Bacterial DNA was isolated from rats' feces samples using Power Fecal® DNA Isolation Kit (MO BIO, Carlsbad, USA) according to the manufacturer's protocol. After being isolated, the DNA concentration was measured with NanoDrop 2000 spectrophotometer (Thermo Fisher Scientific, Wilmington, USA).

\section{7. cDNA synthesis}

The RNA samples were converted to cDNA using Revert Aid First Strand cDNA Synthesis Kit. Samples of RNA with the concentration of $25 \mathrm{ng} / \mu \mathrm{L}$ were used as a template to create cDNA with the final volume $20 \mu \mathrm{L}$. The mixture containing $0.05 \mu \mathrm{g} / \mu \mathrm{L}$ control GAPDH, $0.5 \mu \mathrm{g} / \mu \mathrm{L}$ oligo-dT, $200 \mathrm{U} / \mu \mathrm{L}$ Revertaid Reverse Transcriptase, $20 \mathrm{U} / \mu \mathrm{L}$ Ribolock RNase inhibitor, $5 \mathrm{X}$ reaction buffer, $10 \mathrm{mM}$ dNTP mix, and $10 \mu \mathrm{M}$ of each primer. The PCR reaction was carried out as followed: $25^{\circ} \mathrm{C}$ for 5 minutes, $42^{\circ} \mathrm{C}$ for 1 hour, and $70^{\circ} \mathrm{C}$ for 5 minutes.

\subsection{Quantitative PCR}

Gene expressions were analyzed by quantitative polymerase chain reaction (qPCR) method using Applied Biosystems StepOnePlus Real Time PCR (Thermo Fisher, Waltham, Massachusetts, United States). The DNA samples from cDNA synthesis were amplified in a final volume of $20 \mu \mathrm{L}$ containing $2 \mathrm{X}$ SYBR Green mastermix and $0.8 \mu \mathrm{M}$ of each primer. The primers used for qPCR were F:5-CCACGCTCTTCTGTCTACTG-3; R:5-GCTACGGGCTTGTCACTCGA-3 for tnf- $\alpha$ (Lv et al., 2012), F:5-AGCGTA GAAGGGGACTTGGT-3; R:5-ATGGTGGTCGACTTTCCATC-3 for fabp4 (Eckertova et al., 2011), F:5AGGCAGCTCAGTCCCATAAA-3; R:5-GTAG GTGCCTTCTCGCTGTC-3 for alt2 (Techapiesancharoenkij et al., 2014), F:5GCCGCATGTCAGACCTTCC-3; R:5GACCTGTCCACCAAGTTCTCG-3 for fto (Wang et al., 2011), F:5-AGACCTCTATGCCAACACAGTGC-3; R:5-GAGCCACCAATCCACACAGAGT-3 for $\beta$-actin (Wang et al., 2011), F:5CAAGGTCATCCATGACAACTTTG-3; R:5GTCCACCACCCTGTTGCTGTAG-3 for gapdh (Wang et al., 2015). The data acquired would be analyzed using comparative $\mathrm{C}_{\mathrm{T}}$ method $\left(2^{-\Delta \Delta C_{T}}\right.$ ).The qPCR program was carried out as followed: pre-denaturation at $95^{\circ} \mathrm{C}$ for 2 minutes, denaturation at $95^{\circ} \mathrm{C}$ for 20 seconds, annealing at $64^{\circ} \mathrm{C}$ for 45 seconds, elongation at $72^{\circ} \mathrm{C}$ for 30 seconds, with the total number of cycles was 45 for tnf- $\alpha$; pre-denaturation at $95^{\circ} \mathrm{C}$ for 3 minutes, 30 seconds at $95^{\circ} \mathrm{C}, 30$ seconds at $65^{\circ} \mathrm{C}$ ( gapdh) or $63^{\circ} \mathrm{C} \mathrm{(fabp4} \mathrm{and}$ alt2) and 30 seconds at $72^{\circ} \mathrm{C}$ with the total number of cycles was 45 ; pre-denaturation at $95^{\circ} \mathrm{C}$ for 2 minutes, denaturation at $95^{\circ} \mathrm{C}$ for 15 seconds, annealing with specific temperature of each primer for 30 seconds, and elongation at $72^{\circ} \mathrm{C}$ for 30 seconds with the total number of cycles was 40 for fto, $\beta$-actin $(\beta)$, and $C$. coccoides groups. The specific annealing temperature for each primer were $64^{\circ} \mathrm{C}$ for thf- $\alpha, 72^{\circ} \mathrm{C}$ for fabp $4,72^{\circ} \mathrm{C}$ for alt $2,55^{\circ} \mathrm{C}$ for $f$ to, $59^{\circ} \mathrm{C}$ for $\beta$-actin, and $65^{\circ} \mathrm{C}$ for gapdh.

\subsection{Specific bacteria sequence amplification}

From total bacteria DNA samples, specific bacteria sequence would be amplified with specific 
primer according to its $\mathrm{Tm}$ in Polymerase Chain Reaction (PCR). The Reaction was prepared with 12.5 $\mu \mathrm{L}$ as total volume reaction and consisted of $6.25 \mu \mathrm{L}$ GoTaq Green ${ }^{\circledR}$ Master Mix (Promega, Madison, USA), $2 \mu \mathrm{L}$ template DNA feces, $0.5 \mu \mathrm{L}$ for each forward and reverse primer $(10 \mu \mu) \quad$ Ccoc F:5AAATGACGGTACCTGACTAA-3; R: 5CTTTGAGTTTCATTCTTGCGAA -3 for $C$. coccoides (Kurakawa et al., 2015), and $3.25 \mu \mathrm{L}$ nuclease free water. G-Storm GS482 Thermal Cycler (G-Storm, Somerton, Somerset, United Kingdom) instrument was used for this reaction with initial condition $94^{\circ} \mathrm{C}$ for 5 minutes, denaturation $94^{\circ} \mathrm{C}$ for 30 seconds, annealing condition $55^{\circ} \mathrm{C}$ for $C c o c$ according to $\mathrm{Tm}$ primer, extension $72^{\circ} \mathrm{C}$ for 1 minutes, and post elongation $72^{\circ} \mathrm{C}$ for 10 minutes. This reaction ran for 30 cycles. Afterwards, the amplification product was purified and verified. Verification was done by electrophoresis with $1 \%$ agarose gel in $1 \mathrm{X}$ buffer TAE, $100 \mathrm{~V}, 35$ minutes. The agarose gel was stained with ethidium bromide (EtBr) in dark condition for 15 minutes followed by destaining with aquades for 5 minutes. Finally, the PCR product could be seen in the agarose gel with UVtransilluminator.

\subsection{Plasmid construction}

Plasmid construction method was done to make a standard curve for calculating the amount of specific bacteria in fecal samples. After getting specific bacteria sequence from PCR, the next step was ligation. Amplification product was purified with QIAquick Gel Extraction Kit (QIAGEN, Hilden, Germany) before being ligated to pGEM-T Easy Vector Systems I (Promega, Madison, USA). On the ligation step, we used $20 \mu \mathrm{L}$ ligation reaction consisted of $1 \mu \mathrm{L}$ pGEM-T-Easy (Promega, Madison, USA), $1 \mu \mathrm{L}$ T4 DNA ligase (Promega, Madison, USA), $2 \mu \mathrm{L}$ buffer ligase $2 \mathrm{X}$ (Promega, Madison, USA), $5 \mu \mathrm{L}$ purified amplification product, and nuclease free water. The ligation reaction was incubated at $4{ }^{\circ} \mathrm{C}$ for 16 hours. After ligation process, the ligated plasmid was transformed into Escherichia coli DH5a. Transformation was divided into two main steps, making competent cells and transformation. Competent cells were made using magnesium chloride $\left(\mathrm{MgCl}_{2}\right)$ and calcium chloride $\left(\mathrm{CaCl}_{2}\right)$ salts, while transformation process was done with heat shock method in $42^{\circ} \mathrm{C}$ for one minute. The transformed cells then were grown on Luria Agar media contained ampicillin $(50 \mu \mathrm{g} / \mathrm{mL})$ and X-Gal $(40 \mu \mathrm{g} / \mathrm{mL})$ for blue-white screening process. All media were incubated at $37^{\circ} \mathrm{C}$ overnight. Grown white colony bacteria indicated successful ligation process to vector.
To clone the plasmid, the white colony was refreshed to Luria Agar media contained ampicillin in $37^{\circ} \mathrm{C}$ for 12 until 16 hours. The plasmid from refreshed bacteria was isolated with Wizard ${ }^{\circledR}$ Plus SV Minipreps DNA Purification System (Promega, Madison, USA).

\subsection{Verification}

To verify the isolated plasmid, plasmid was quantified with NanoDrop 2000 spectrophotometer (Thermo Fisher Scientific, Wilmington, USA). Then, plasmid was amplified with M13 forward-reverse primer. These amplification products were sequenced and checked with basic local alignment searching tools (BLAST) to make sure the plasmid was inserted into the right DNA template.

\subsection{Standard curve construction}

Standard curve was used to calculate the amount of specific bacteria in feces sample. The constructed plasmid was then diluted into five different concentrations from $5 \times 10^{1}$ to $5 \times 10^{5}$ to make the standard curve. Amount of specific bacteria DNA copy number was measured with the formula following according to Avogadro number and molecular mass for each nucleotide base as in.

$$
\text { number of copies }=\frac{\text { amount of DNA }(n g) \times 6.022 \times 10^{25}}{\text { DNA lenght }(b p) \times 1 \times 10^{9} \times 650}
$$

\subsection{Data analysis}

The amount of specific bacteria DNA copy number was converted into amount of specific bacteria by dividing the specific bacteria DNA copy number with operon number from 16S rRNA gene database. Livak or comparative $\mathrm{Ct}$ Mean method was used for gene expression analysis.

\subsection{Statistical analysis}

Statistical analysis was performed using SPSS version 21.0 (IBM, Armonk, New York, United States). Shapiro-Wilk test was performed for normality test. Comparisons between groups were analyzed using OneWay ANOVA and posthoc Tukey test. For the comparison of blood glucose measurement within group, statistical analysis was performed using repeated measure ANOVA. Significance was assigned at P-value of $<0.05$.

\section{Results}

\subsection{Sucrose increased the rats' body and liver weight}


The body weight of each rat was measured every week during the seven weeks of treatment as shown in Figure 1A and 1B; while liver weight was measured after rats were sacrificed and the livers were excised at the end of the experiment as shown in Figure 1C. From the data, it was clear that body and liver weight of sucrose group were significantly higher $(p<0.05)$ compared to control and aspartame groups. From Figure 1A, the significant difference in body weight was started to appear from week 3 , and continued to show the similar trend up to day 40. Meanwhile, the body weight during the treatment and on day 40; as well as liver weight in aspartame group on day 40 did not differ with the control group.

\subsection{Aspartame maintained the blood glucose level}

Blood glucose level for each rat was measured before the treatment was administered, in which all the rats had comparable basal blood glucose values (data not shown). Based on Figure 2, at the end of treatment (day 40), the group which received sucrose had a significantly greater blood glucose value $(p<0.05)$ compared to aspartame and control groups.

\subsection{Aspartame did not affect the fto gene expression}

Figure 3 demonstrated that, at the end of the experiment (day 40), sucrose treatment decreased the expression of fto gene; yet, the level of fto gene in aspartame group was comparable to the control group.

\subsection{Aspartame showed lower expression of several metabolic syndrome-related markers}

In this study, expression of several genes that was considered as metabolic syndrome-related biomarkers was measured. The target genes were fabp 4 gene from adipose tissue as shown in Figure 4A, alt 2 from liver tissue in Figure 4B, and tnf- $\alpha$ gene from blood samples in Figure 4C. Our data demonstrated that sucrose treatment gave the highest increase in expression level of those genes; while aspartame treatment showed much lower values. No significant difference was found between groups. However, these data showed a similar trend with the body weight and liver weight data.

\subsection{Population of C. coccoides group was greatly decreased by sucrose administration}

The difference between $C$. coccoides population between day 0 up to day 40 was observed. Our data showed that, after 40 weeks of administration, sucrose contributed a significant reduction in $C$. coccoides population $(\mathrm{p}<0.05)$ as shown in Figure 5. Meanwhile, the $C$. coccoides population in aspartame group was not significantly affected compared to the control group.

\section{Discussion}

Our results clearly demonstrated that rats which were given sucrose treatment had a significantly higher body and liver weight, compared to the aspartame and control groups. Sucrose could induce body weight gain because it induced a higher amount of calorie intake to reach the same sweetness level as aspartame did. Each gram of both ingredients gave $4 \mathrm{kcal}$ of energy. Yet, aspartame had 200 times higher sweetness level; contributing only $0.02 \mathrm{kcal}$ for the same sweetness level with sucrose in food products (Mair et al., 2005; Magnuson et al., 2007). This might explain the major difference in body weight between rats in sucrose and aspartame group; in which the major difference started to appear after 3 weeks of

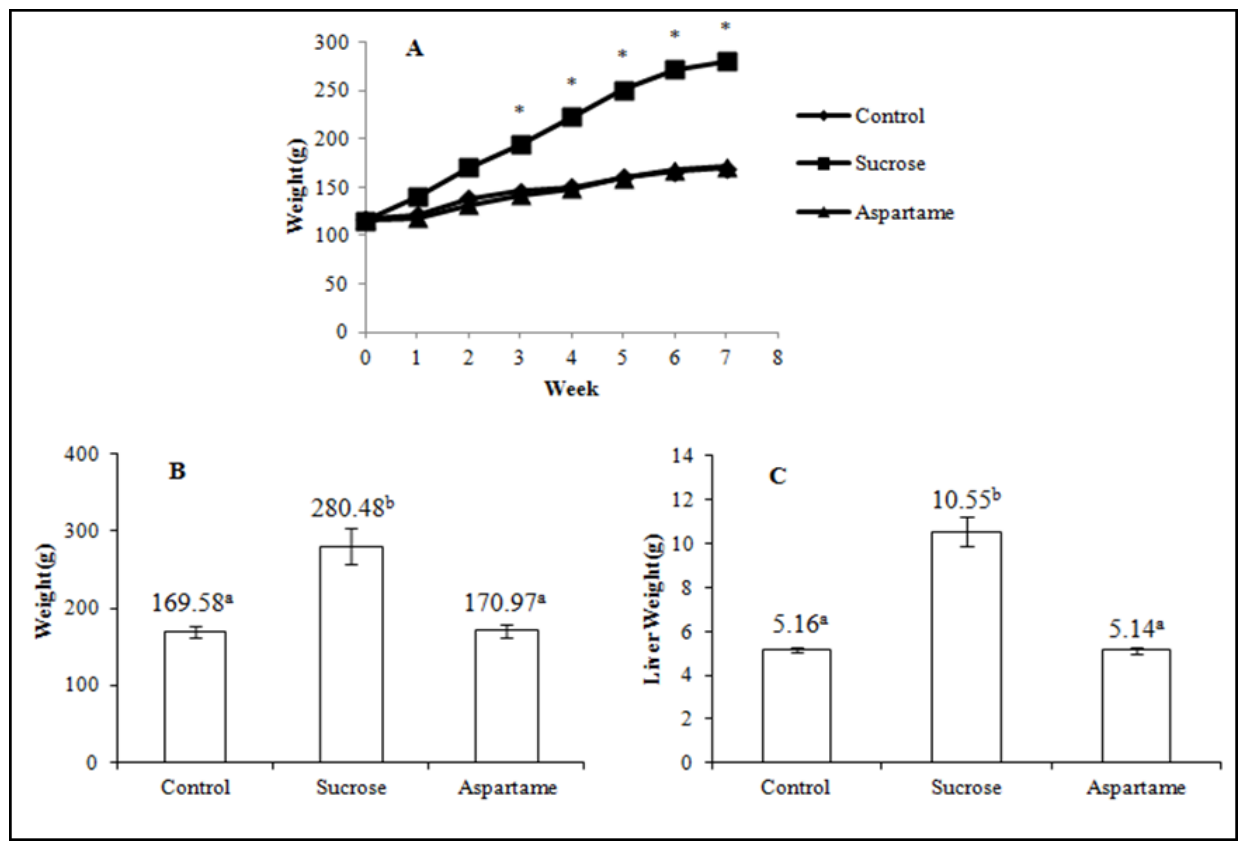

Figure 1. Body weight measurements of control, sucrose, and aspartame group during 7 weeks of treatment (A) and at the end of the treatment (day 40) (B). Liver weight measurement of three groups at the end of the treatment (day 40) (C). Data are expressed as mean \pm SE $(n=5)$. Values with different superscript or (*) superscript were significantly different $(\mathrm{p}<0.05)$ 


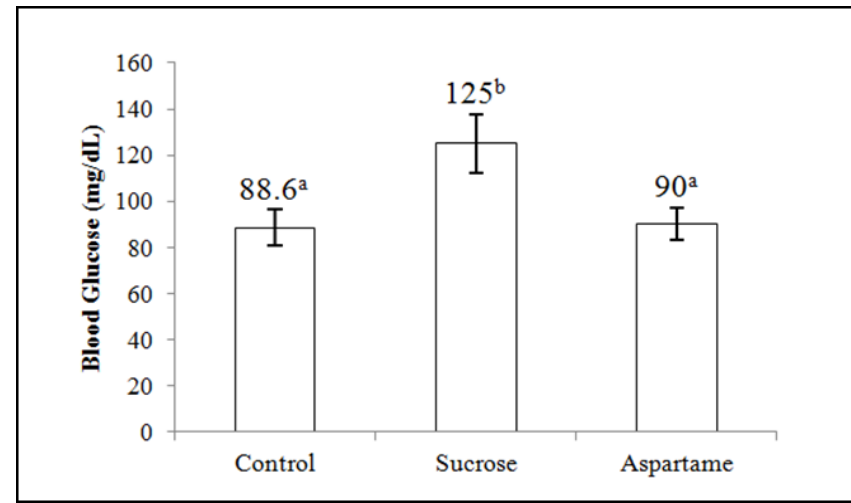

Figure 2. Blood glucose measurement at the end of the treatment (day 40). Data are expressed as mean $\pm \mathrm{SE}$ $(n=5)$. Values with different superscript were significantly different between groups $(\mathrm{p}<0.05)$

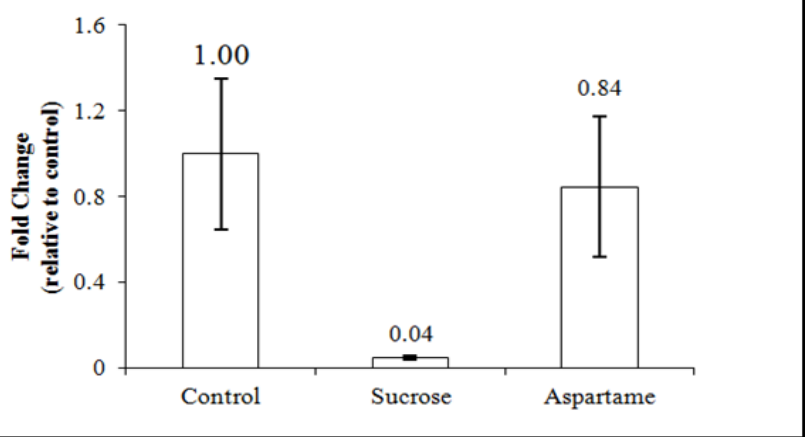

Figure 3. Expression of fto gene from the cerebellum. Data are expressed as mean $\pm \mathrm{SE}(\mathrm{n}=5)$. No values were significantly different $(\mathrm{p}<0.05)$

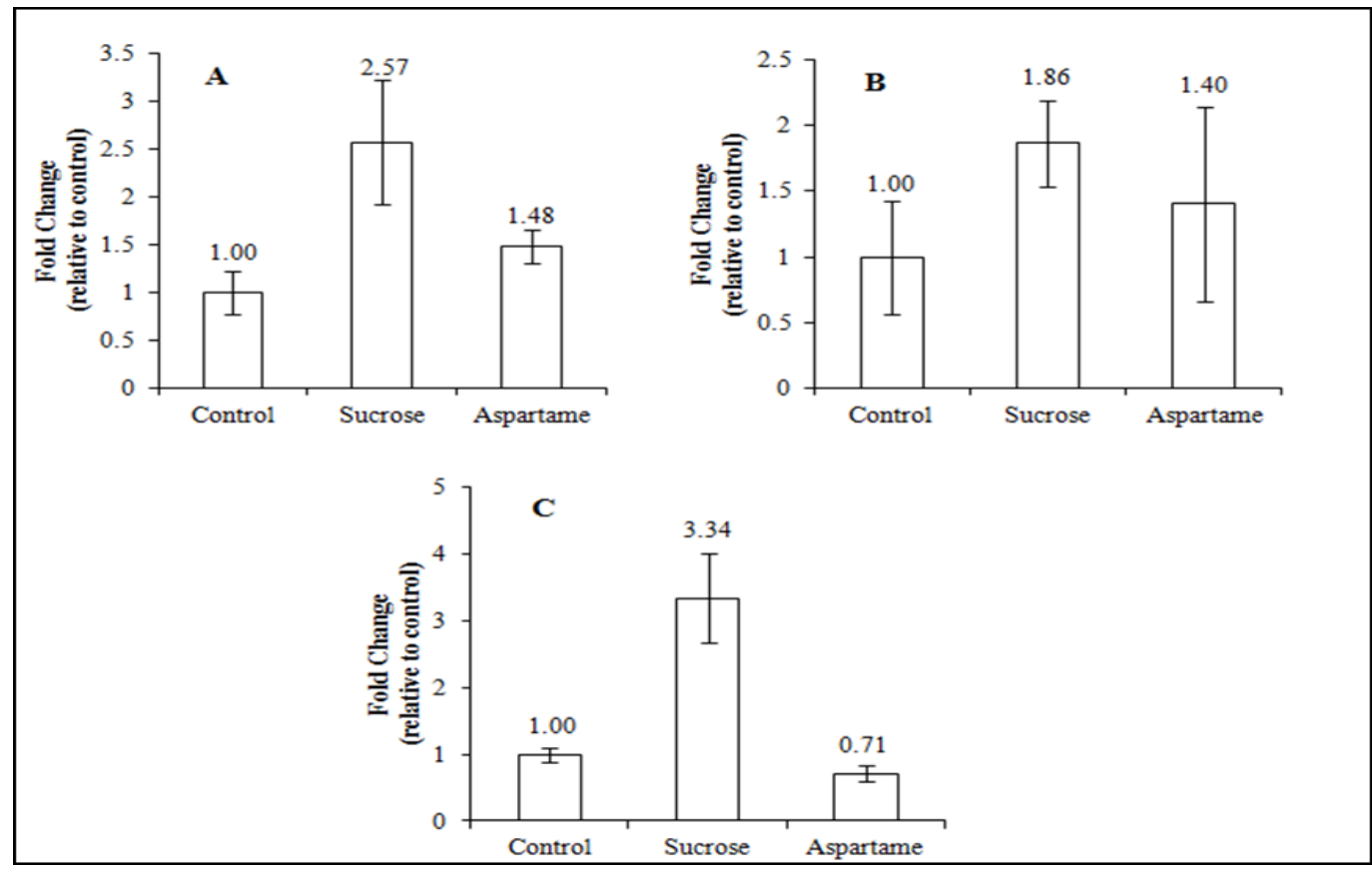

Figure 4. Gene expression measurement from adipose tissue, liver, and blood samples. Expression of fabp 4 gene from adipose tissue (A). Expression of alt2 from liver tissue (B). Expression of $t n f-\alpha$ gene from blood (C). Data are expressed as means $\pm \mathrm{SE}$ $(\mathrm{n}=5)$. No values were significantly different $(\mathrm{p}<0.05)$

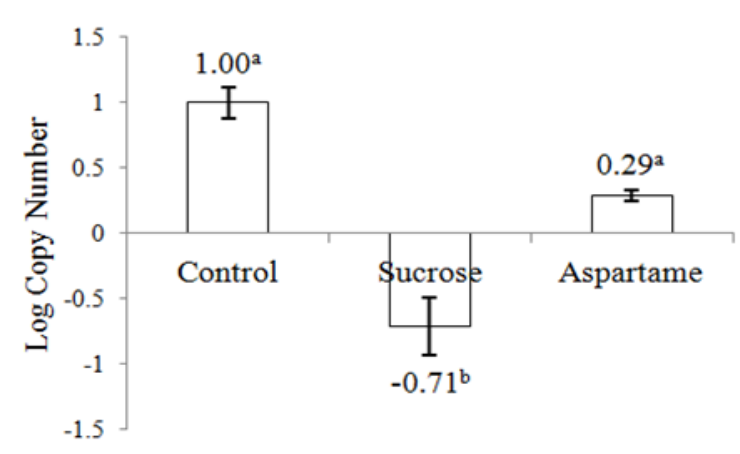

Figure 5. Difference of $C$. coccoides number in control, sugar and aspartame group between day 40 and day 0 ( $\mathrm{n}=5$ in each group). Values with different superscript were significantly different $(\mathrm{p}<0.05)$ treatment, and continued to be significantly different until the end of experimental period (day 40).

Non-alcoholic fatty liver disease (NAFLD) is a liver disease characterized by excessive fat accumulation in liver (triglyceride $>5 \%$ hepatocyte). Fatty liver or steatosis is the first stage of NAFLD. It could progress into more severe condition, such as non-alcoholic steatohepatitis (NASH) and fibrosis, cirrhosis, and sometimes, carcinoma (Serfaty and Lemoine 2008). Based on NAFLD characteristic, enlargement of liver could be one of the metabolic disorder symptoms. Our experiment showed that the average liver weight in sucrose group was higher than aspartame and control group. In sucrose-treated group, rats were given high concentration of sucrose $(30 \% \mathrm{w} / \mathrm{v})$. The excessive 
calorie and sucrose intake caused insulin resistant state; in which the adipocyte lipolysis increased independently from nutritional status, leading to abundant free fatty acids in plasma pool. This caused increased fatty acid uptake and fat accumulation in liver (Bechmann et al., 2012); and finally resulted in liver enlargement, as observed in our experimental data. Meanwhile, the liver weight in groups treated with aspartame $(0.15 \% \mathrm{w} / \mathrm{v}) \mathrm{did}$ not show any notable difference with the control group.

High blood glucose level (hyperglycemia) has been long associated with the higher probability of type 2 diabetes mellitus development. The blood glucose value is widely used to classify subjects into several states: normal $(<100 \mathrm{mg} / \mathrm{dL})$, pre-diabetes (between $100-125$ $\mathrm{mg} / \mathrm{dL}$ ), and diabetes $(>125 \mathrm{mg} / \mathrm{dL}$ ) (Genuth et al., 2003). This study showed a significantly higher blood glucose level in sucrose-treated rats $(125 \mathrm{mg} / \mathrm{dL})$, compared to the aspartame $(90 \mathrm{mg} / \mathrm{dL})$ and control $(88.6$ $\mathrm{mg} / \mathrm{dL}$ ) group on day 40. Moreover, all rats belonged to sucrose group could be classified as "pre-diabetes" at the end of the treatment, correlated to the blood glucose value states. Thus, these results confirmed previous studies about the beneficial effect of aspartame in maintaining blood glucose better than sucrose did.

Several studies remarked the possibility of aspartame in inducing craving behavior (Yang 2010). A study demonstrated that fto gene expression in rats' hypothalamus was up-regulated when they were fasting (Vujovic et al., 2013). Moreover, the fto expression was correlated positively with the level of ghrelin as hungerinducing hormone (Karra et al., 2013). Therefore, in this experiment, we investigated the level of fto gene expression in cerebellum tissue as a marker to identify the craving or hunger level of the experimental rats.

Our results showed that sucrose reduced the level of fto gene expression; indicating sucrose did deliver the satiety effect to the experimental rats. The expression of fto gene in aspartame-treated rats was higher than sucrose group, but it was still a bit lower compared to the control group. This implying that, although aspartame did not give satiety effect as high as the sucrose did; yet, aspartame did not induce hunger in the rats either. So, previous studies which suggested that aspartame induced craving was not proven in this research.

As already mentioned above, sucrose consumption was likely to contribute extra calories compared to aspartame. These excessive calories would be stored as fat via a process called lipogenesis. Lipogenesis mechanism involved several regulatory proteins, like sterol regulatory element-binding protein 1 (SREBP-1) and carbohydrate-responsive element-binding protein (ChREBP), as the major transcriptional regulators of lipogenesis in the liver. SREBP isoforms (SREBP-1a, SREBP-1c and SREBP2) and ChREBP were engaged in both transcriptional and post-transcriptional mechanisms. The monomers of sucrose acted as substrates for fatty acid synthesis and influenced the transcriptional regulation of lipogenesis. Fructose induced SREBP-1 and ChREBP, while glucose only induced ChREBP (Moore et al., 2014). Studies reported that the expression of fabp 4 was high when lipogenesis took place; since FABP4 would act as lipid transporter which accommodates the fatty acid transfer (Serr et al., 2009). Therefore, in this experiment, we hypothesized that sucrose treatment would raise the expression of fabp 4 in adipose tissue.

Our result demonstrated a similar trend; in which fabp4 gene expression was almost three-times higher compared to the control group. Meanwhile, the expression of the fabp 4 gene in aspartame-treated mice was about half the sucrose group. The higher level of fabp4 gene expression might induce a higher level of FABP4 protein, which accommodated the fatty acid transport from and into the adipose tissues; and led to stimulation of higher adipocyte lipogenesis. Furthermore, a lower fabp 4 gene expression in aspartame group suggested that aspartame induced lipogenesis in a minor level compared to sucrose. The level of fabp 4 gene expression was also positively correlated with inflammation marker (Terra et al., 2014); proposing a higher inflammatory state was induced by sucrose compared to the two other treatments.

The expression of alt 2 gene in liver tissue was also observed. The sucrose group showed the highest expression level, which was almost two-times fold compared to control. Meanwhile, the expression of alt 2 gene in aspartame-treated mice was lower compared to the sucrose. High concentration of sucrose might lead to excessive energy intake and insulin resistance in rats. As described above, insulin resistant state induced the upregulation of gluconeogenic enzymes, such as ALT (Saltiel and Kahn 2001). This could explain the upregulation of alt 2 expression in sucrose group. Taken together with the blood glucose value, the data from alt 2 gene expression suggested the beneficial effect of aspartame in maintaining blood glucose level.

Excessive sucrose consumption had been stated to elevate the tnf- $\alpha$ gene expression in human blood; of which was associated with metabolic syndrome development (Kempf et al., 2007). In our experiment, the 
highest $\operatorname{tnf}-\alpha$ gene expression was observed in sucrosetreated mice; while aspartame seemed to lower tnf- $\alpha$ expression compared to sucrose, and even the control group. This result agreed previous study by Sharma et al. (2005) which showed aspartame had a possible antiinflammatory effect based on the paw volume measurement (Sharma et al., 2005). In overall, our result confirmed previous data proclaiming that TNF- $\alpha$ concentration would elevate when sucrose consumption was high; yet, sucrose replacement with aspartame would lower the TNF- $\alpha$ concentration (Kempf et al., 2007; De Koning et al., 2012; Jung and Choi 2014; Choudhary and Devi 2015). However, further studies are needed to understand the detailed mechanism.

In this study, the effect of sucrose and aspartame on C. coccoides population was also monitored. The sucrose treatment significantly decreased the $C$. coccoides population at the end of the experimental period; while the variation in aspartame group was not notably different compared to the control group. These results, taken together with the body weight data, agreed on the previous research about $C$. coccoides group; which stated the excessive calorie consumption in obese people could suppress the growth of $C$. coccoides (Schwiertz et al., 2009). However, although the number was not significantly different; a slight reduction in $C$. coccoides population was observed in aspartame group. Interestingly, the average body weight values between aspartame and control group in day 40 was actually comparable. These data corresponded to the study from Noble et al. (2017) which stated that dietary pattern altered the gut microbiota composition, independently of obesity status.

To our knowledge, there was still limited information about the effects of sucrose and artificial sweetener consumption using metabolic syndromerelated gene expression and gut microbiota population, as the potential biomarkers. Along with the increment of worldwide diabetes prevalence, this might lead to the increase artificial sweetener consumption; these research topics deserve further investigations. Furthermore, although this study implied that the artificial sweetener seemed to offer more beneficial effects compared to sucrose; more studies are still needed to understand the detailed mechanisms.

\section{Conclusion}

In summary, this study confirmed that aspartame had more beneficial effects in maintaining body weight and blood glucose level, compared to sucrose consumption. Besides, aspartame group also demonstrated a less fat accumulation in liver; and did not show elevation in craving behavior as well. Several markers related with inflammation, lipogenesis and insulin resistance were also lower in aspartame group. Moreover, although it was proven that dietary pattern did alter the gut microbiota population; our data indicated that sucrose consumption might lead to a more severe dysbiosis. Taken together, this study concluded that aspartame might be considered as a safe and favorable as sugar substitute; especially in the era of raise of diabetes prevalence worldwide.

\section{Acknowledgements}

This research was funded by PT. Nutrifood Indonesia. The authors would like to thank Ritchie Rahardja for literature review and data analysis.

\section{References}

Abhilash, M., Paul, M.V.S., Varghese, M.V. and Nair, R.H. (2011). Effect of long term intake of aspartame on antioxidant defense status in liver. Food and Chemical Toxicology, 49(6),1203-1207. doi:10.1016/j.fct.2011.02.019.

Bechmann, L.P., Hannivoort, R.A., Gerken, G., Hotamisligil, G.S., Trauner, M. and Canbay, A. (2012). The interaction of hepatic lipid and glucose metabolism in liver diseases. Journal of Hepatology, 56(4), 952-964. doi:10.1016/j.jhep.2011.08.025.

Choudhary, A.K. and Devi, R.S. (2015). Longer period of oral administration of aspartame on cytokine response in Wistar albino rats. Endocrinologia $Y$ Nutricion, 62(3), 114-122. doi:10.1016/ j.endonu.2014.11.004.

Collison, K.S., Makhoul, N.J., Zaidi, M.Z., Saleh, S.M., Andres, B., Inglis, A., Al-Rabiah, R. and AlMohannna, FA. (2012). Gender dimorphism in aspartame-induced impairment of spatial cognition and insulin sensitivity. PLoS One, 7(4), e31570. doi:10.1371/journal.pone.0031570.

De Koning, L., Malik, V.S., Kellogg, M.D., Rimm, E.B., Willett, W.C. and Hu, F.B. (2012). Sweetened beverage consumption, incident coronary heart disease, and biomarkers of risk in men. Circulation, 125(14), 1735-1741. doi: 10.1161/CIRCULATIONAHA.112. 123208.

Eckertova, M., Ondrejcakova, M., Krskova, K., Zorad, S. and Jezova, D. (2011). Subchronic treatment of rats with oxytocin results in improved adipocyte 
differentiation and increased gene expression of factors involved in adipogenesis. British Journal of Pharmacology, 162(2), 452-463. doi: 10.1111/ j.1476-5381.2010.01037.x.

Feijo, F.M., Ballard, C.R., Foletto, K.C., Batista, B.A.M., Neves, A.M., Ribeiro, M.F.M. and Bertoluci, M.C. (2013). Saccharin and aspartame, compared with sucrose, induce greater weight gain in adult Wistar rats, at similar total caloric intake levels. Appetite, 60, 203-207. doi:10.1016/ j.appet.2012.10.009.

Fowler, S.P.G., Williams, K. and Hazuda, H.P. (2015). Diet soda intake is associated with long-term increases in waist circumference in a bi-ethnic cohort of older adults: the San Antonio longitudinal study of aging. Journal of American Geriatrics Society, 63(4), 708-715. doi:10.1111/jgs.13376.

Furuhashi, M., Saitoh, S., Shimamoto, K. and Miura, T. (2014). Fatty acid-binding protein 4 (FABP4): pathophysiological insights and potent clinical biomarker of metabolic and cardiovascular diseases. Clinical Medicine Insights: Cardiology, 8(S3), 2333. doi:10.4137/CMC.S17067.

Genuth, S., Alberti, K.G., Bennett, P., Buse, J., DeFronzo, R., Kahn, R., Kitzmiller, J., Knowler, W.C., Lebovitz, H., Lernmark, A. and Nathan, D. (2003). Follow-up report on the diagnosis of diabetes mellitus. Diabetes Care, 26(11), 3160-8. doi:10.2337/diacare.26.11.3160.

Jung, U.J. and Choi, M.S. (2014). Obesity and its metabolic complications: The role of adipokines and the relationship between obesity, inflammation, insulin resistance, dyslipidemia and nonalcoholic fatty liver disease. International Journal of Molecular Science, 15(4), 6184-6223. doi: 10.3390/ ijms15046184.

Karra, E., O’Daly, O.G., Choudhury, A.I., Yousseif, A., Millership, S., Neary, M.T., Scott, W.R., Chandarana, K., Manning, S., Hess, M.E. and Iwakura, H. (2013). A link between FTO, ghrelin, and impaired brain food-cue responsivity. Journal of Clinical Investigation, 123(8), 3539-3551. doi:10.1172/JCI44403.

Kempf, K., Rose, B., Herder, C., Haastert, B., FusbahnLaufenburg, A., Reifferscheid, A., Scherbaum, W.A., Kolb, H. and Martin, S. (2007). The metabolic syndrome sensitizes leukocytes for glucose-induced immune gene expression. Journal of Molecular Medicine, 85(4), 389-396. doi:10.1007/s00109-006-0132-7.
Kurakawa, T., Ogata, K., Matsuda, K., Tsuji, H., Kubota, H., Takada, T., Kado, Y., Asahara, T., Takahashi, T. and Nomoto, K. (2015). Diversity of intestinal Clostridium coccoides group in the Japanese population, as demonstrated by reverse transcription-quantitative PCR. PLoS ONE ,10(5), 1 -19. doi:10.1371/journal.pone.0126226.

Lopetuso, L.R., Scaldaferri, F., Petito, V. and Gasbarrini, A. (2013). Commensal Clostridia: leading players in the maintenance of gut homeostasis. Gut Pathogens, 5(23), 1-8. doi: 10.1186/1757-4749-5-23.

Lv, X., Li, Q., Wu, S., Sun, J., Zhang, M. and Chen, Y.J. (2012). Psychological stress alters the ultrastructure and increases IL-1 $\beta$ and TNF- $\alpha$ in mandibular condylar cartilage. Brazilian Journal of Medical and Biological Research, 45(10), 968-976. doi:10.1590/S0100-879X2012007500 102.

Magnuson, B.A., Burdock, G.A., Doull, J., Kroes, R.M., Marsh, G.M., Pariza, M.W., Spencer, P.S., Waddell, W.J., Walker, R. and Williams. (2007). Aspartame: a safety evaluation based on current use levels, regulations and toxicological and epidemiological studies. Critical Reviews in Toxicology, 37(8), 629727. doi:10.1080/10408440701516184.

Mair, W., Piper, M.D.W. and Partridge, L. (2005). Calories do not explain extension of life span by dietary restriction in Drosophila. PLoS Biology, 3 (7), 1305-1311.doi:10.1371/journal.pbio.0030223.

Moore, J.B., Gunn, P.J. and Fielding, B.A. (2014). The role of dietary sugars and de novo lipogenesis in non-alcoholic fatty liver disease. Nutrients, 6(12), 5679-5703. doi:10.3390/nu6125679.

Noble, E.E., Hsu, T.M., Jones, R.B., Fodor, A.A., Goran, M.I. and Kanoski, S.E. (2017). Early-life sugar consumption affects the rat microbiome independently of obesity. The Journal of Nutrition, 147(1), 20-28. doi: 10.3945/jn.116.238816

Palmnas, M.S., Cowan, T.E., Bomhof, M.R., Su, J., Reimer, R.A., Vogel, H.J., Hittel, D.S. and Shearer, J. (2014). Low-dose aspartame consumption differentially affects gut microbiota-host metabolic interactions in the diet-induced obese rat. PLoS ONE, 9(10), e109841. doi:10.1371/ journal.pone.0109841.

Saltiel, A.R. and Kahn, C.R. (2001). Insulin signalling and the regulation of glucose and lipid metabolism. Nature, 414(6865), 799-806. doi:10.1038/414799a.

Schwiertz, A., Taras, D., Schafer, K., Beijer, S., Bos, N.A., Donus, C. and Hardt, P.D. (2009). Microbiota 
and SCFA in lean and overweight healthy subjects. Obesity, 18(1), 190-196. doi:10.1038/oby .2009.167

Serr, J., Suh, Y. and Lee, K. (2009). Regulation of adipose triglyceride lipase by fasting and refeeding in avian species. Poultry Science, 88(12), 25852591. doi:10.3382/ps.2009-00265.

Serfaty, L. and Lemoine, M. (2008). Definition and natural history of metabolic steatosis: clinical aspects of NAFLD, NASH, and cirrhosis. Diabetes and Metabolism Journal, 34(6), 634-637. doi:10.1016/S1262-3636(08)74597-X.

Sharma, S., Jain, N.K. and Kulkarni, S.K. (2005). Possible analgesic and anti-inflammatory interactions of aspartame with opioids and NSAIDs. Indian Journal of Experimental Biology, 43(6), 498.

Simon, B.R., Parlee, S.D., Learman, B.S., Mori, H., Scheller, E.L., Cawthorn, W.P., Ning, X., Gallagher, K., Tyrberg, B., Assadi-Porter, F.M. and Evans, C.R. (2013). Artificial sweeteners stimulate adipogenesis and suppress lipolysis independently of sweet taste receptors. Journal of Biological Chemistry, 288(45), 32475-32489. doi: 10.1074/ jbc.M113.514034.

Terra, X., Quintero, Y., Auguet, T., Porras, J.A., Hernandez, M., Sabench, F., Aguilar, C., Luna, A.M., del Castillo, D. and Richart, C. (2011). FABP 4 is associated with inflammatory markers and metabolic syndrome in morbidly obese women. European Journal of Endocrinology, 164(4), 539547. doi: 10.1530/EJE-10-1195.

Techapiesancharoenkij, N., Fiala, J.L.A., Navasumrit, P., Croy, R.G., Wogan, G.N., Groopman, J.D., Ruchirawat, M. and Essigmann, J.M. (2014). Sulforaphane, a cancer chemopreventive agent, induces pathways associated with membrane biosynthesis in response to tissue damage by aflatoxin B1. Toxicology and Applied Pharmacology, 282(1), 52-60. doi:10.1016/ j.taap.2014.11.004.

Vujovic, P., Stamenkovic, S., Jasnic, N., Lakic, I., Djurasevic, S.F., Cvijic, G. and Djordjevic, J. (2013). Fasting induced cytoplasmic fto expression in some neurons of rats hypothalamus. PLOS ONE, 8(5), 1-6. doi:10.1371/journal.pone.0063694.

Wang, P., Yang, F.J., Du, H., Guan, Y.F., Xu, T.Y., Xu, X.W., Su, D.F. and Miao, C.Y. (2011). Involvement of leptin receptor long isoform (LepRb)-STAT3 signaling pathway in brain fat mass-and obesityassociated (fto) downregulation during energy restriction. Molecular Medicine, 17(5-6), 523532. doi:10.2119/molmed. 2010.00134.

Wang, X., Zhang, J., Zhou, L., Lu, P., Zheng, Z.G., Sun, W., Wang, J.L., Yang, X.S., Li, X.L., Xia, N. and Zhang, N. (2015). Significance of serum microRNA-21 in diagnosis of hepatocellular carcinoma (HCC): clinical analyses of patients and an HCC rat model. International Journal of Clinical and Experimental Pathology, 8(2), 14661476.

Yang, Q. (2010). Gain weight by “going diet?” artificial sweeteners and the neurobiology of sugar cravings. Yale Journal of Biology and Medicine, 83(2), 101108. 